\title{
Research on the Effect of BMI1 Gene Silencing on the Proliferation Activity of Human Breast Cancer Cells
}

\author{
Penghui Li ${ }^{1, a}$, Dan Liu, ${ }^{2, b}$, Qingbu Mei ${ }^{3, c}$, Xiuhua Wang ${ }^{4, d}$, Yi Zheng ${ }^{5, e}$, \\ Hongming Pan $^{6, f}$ and Lihong Zheng ${ }^{7, g_{*}}$ \\ 1,2,3,4,5,6,7 Qiqihar Medical University, Qiqihar, Heilongjiang, 161006, China

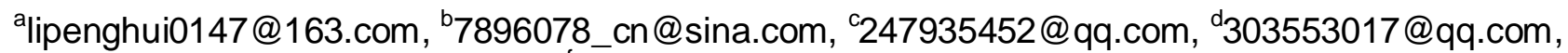 \\ '83964922@qq.com, ${ }^{\mathrm{f}}$. \\ * The Corresponding Author
}

Keywords: BMI1 gene; Breast cancer cell; Gene silencing; Cell apoptosis; Cell proliferation

\begin{abstract}
Objectives: The purpose is to study the effect of proto oncogene BMI1(B-cell specific moloney murine leukemiavirus insertion site 1) gene silencing on the proliferation inhibition and 5-Fu sensitivity of human breast cancer cells through experiment in vitro, and to explore the feasibility of the combined treatment of breast cancer by BMI1 gene silencing and 5-Fu. Methods: The MTT method was used to detect the inhibitory effects of 5-Fu on the proliferation of MCF-7 cells, MCF-7/5-Fu cells and their BMI1 gene silencing cells. Morphological changes of cells in each group stained with acridine orange were observed by fluorescence. The flow cytometry was used to detect the effect of 5-Fu on the apoptosis rate and cell cycle of each group. Results: 5-Fu inhibited the growth of human breast cancer cells, and presented time - dose dependence. At 48h, the IC50 of cells in each group inhibited by 5-Fu was respectively: MCF-7(2.85 \pm 0.21$)$, MCF-7/BMI1si (1.78 \pm 0.25$),$ MCF-7/5-Fu(8.05 \pm 0.38$)$ and MCF-7/5-Fu/BMI1 si (5.65 \pm 0.27$) \mu \mathrm{g} / \mathrm{mL}$. BMI1 gene silencing cells were significantly lower than breast cancer cells(LSD- $t=2.845,3.232$; $P<0.05$ ). If $4 \mu \mathrm{g} / \mathrm{mL} 5-\mathrm{Fu}$ acts on the cells of each group for $48 \mathrm{~h}$, it can induce obvious cell apoptosis features in MCF-7/5-Fu/BMI1si cells. The apoptosis rate of BMI1 gene silencing group was significantly higher than that of breast cancer cells MCF-7 and MCF-7/5-Fu(LSD- $t=7.826$, $8.231 ; P<0.05)$. The cell proportion of BMI1 gene silencing cells in G0/G1 phase also increased significantly (LSD- $t=10.512,8.053 ; P<0.05)$. Conclusions: BMI1 gene silencing can enhance the sensitivity of breast cancer cells to 5 -Fu and promote cell apoptosis.
\end{abstract}

\section{Introduction}

Breast cancer is the malignant tumor that the incidence of women in China is the first and the fatality rate is sixth. In recent years, the incidence has been increasing gradually[1]. One of the important problems in the treatment of breast cancer is the resistance of cancer cells to the cell proliferation inhibition and apoptosis induced by various chemotherapeutic drugs. Therefore, further studying the role of key signaling pathway molecules in the regulation of apoptosis sensitivity of breast cancer cells and finding effective molecular targets will provide useful ideas for the development and clinical treatment of the anticancer drugs of breast cancer.

BMI1, as a proto oncogene, was first found in the mouse lymphoma caused by retrovirus. The human BMI-1 gene is located on the section 13 of the short arm of chromosome 10. Its mRNA is about 3199 bp, including 10 exons, and the sequence has high homology between human and mouse[2,3]. The first evidence to reveal the key role of BMI1 during tumorigenesis is the occurrence process of $\mathrm{T}$ lymphoma and $\mathrm{B}$ lymphoma in transgenic rats. This gene is regulated by the promoter, and the overexpression of the gene leads to abnormal amplification of the lymphocyte[4]. It has been found that the up regulation of BMI1 occurs in many types of tumors, such as lymphoma[5], prostate cancer[6], non-small cell lung cancer[7], colon cancer[8], breast cancer[9], nasopharyngeal carcinoma[10] and so on. Its overexpression is closely related to the occurrence, development and prognosis of tumor, and often indicates poor prognosis. Through the experiment in vitro, this study detected the effect of siRNA expression vector of targeting BMI1 on 
the proliferation and apoptosis of human breast cancer MCF-7 cells and MCF-7/5-Fu cells, in order to provide reference for the diagnosis and treatment of breast cancer.

\section{Materials and Methods}

Materials. The breast cancer cell line MCF- 7 is purchased from the ATCC cell center in the United States. Its 5- fluorouracil resistant cell line $(\mathrm{MCF}-7 / 5-\mathrm{Fu})$ was independently established by our laboratory through $5-\mathrm{Fu}$ induction. The siRNA recombinant expression vector of targeting BMI1 gene was constructed by Wuhan Jin Kairui Biotechnology Co., Ltd. (Positive-sense strand 5'-AGACCAGACCACUACUGAATT-3'; antisense strand 5'-UUCAGUAGUGGUCUGGUCUTG -3'). The experimental group cells MCF-7/BMI1si and MCF-7/5- Fu/BMI1si of the stable transfected recombinant BMI1 gene interfering carrier were obtained by the preliminary experiment of the project group. The experimental reagents, such as trypsin, RPMI1640 culture medium, dimethyl sulfoxide(DMSO), methyl thiazolyl tetrazolium(MTT) and acridine orange dye were all the products of Sigma and Gibco companies. The flow cytometry was BD FACSCalibur, the microplate reader was TECAN SUNRISE, and the fluorescence microscope was Olympus.

Methods. The first is the cell culture. Under the conditions of $5 \% \mathrm{CO}_{2}, 37^{\circ} \mathrm{C}$ and saturated humidity, the cells were cultured in the RPMI1640 complete culture medium containing $10 \%$ fetal bovine serum.

The second is to use MTT method to detect the effect of BMI1 gene silencing on the proliferation activity of breast cancer cells. The cells with the density of $5 \times 10^{3} /$ hole of each group were inoculated to the 96 -well plate. The total amount of cell suspension per hole was $200 \mu \mathrm{L}$. After $24 \mathrm{~h}$ of culture in normal condition, the chemotherapy drug 5-Fu was added. Seven concentration gradients of $0.5,1,2,4,8,16$ and $32 \mu \mathrm{g} / \mathrm{mL}$ were set. At the same time, the cell control group (without drugs) and blank control group (adding $200 \mu \mathrm{L}$ complete medium) were set up, and each group had 3 repetitions. It continued to be cultured in a incubator of $5 \% \mathrm{CO}_{2}$ at $37^{\circ} \mathrm{C}$. after culturing of $48 \mathrm{~h}, 20 \mu \mathrm{L} 5 \mathrm{mg} / \mathrm{mL}$ MTT dye liquor was added to each hole. After culturing $4 \mathrm{~h}$ in normal conditions, the culture solution was removed and $200 \mu \mathrm{L}$ DMSO was added to each hole. Oscillating $10 \mathrm{~min}$ under light avoiding condition. The microplate reader was used to determine the OD value(A value) under the condition that the wavelength of each hole was $492 \mathrm{~nm}$. The reference wavelength was set to $630 \mathrm{~nm}$. The blank group OD $<0.005$ and the experimental group OD $>0.1$ were the effective experiments. The inhibition ratio of cell proliferation of cells of each group was calculated under the action of 5-Fu. Cell inhibition rate $=(\mathrm{OD}$ value of cell control group $-\mathrm{OD}$ value of experimental group) / (OD value of cell control group -OD value of blank control group) $\times 100 \%$. The inhibition curve of cell proliferation was plotted and the median inhibitory concentration (IC50) of the drug was calculated by Bliss.

The third is to use fluorescence microscope to detect the situation of BMI 1 gene silencing promoting the apoptosis of breast cancer cells. The cells with good growth state of each group were inoculated with $1 \times 10^{5} / \mathrm{mL}$ and cultured. When the cells grew to the logarithmic growth period, 5-Fu (Concentration was determined by the IC50 detection results) was added, and then they continue to be cultured in the incubator of $5 \% \mathrm{CO}_{2}$ at $37^{\circ} \mathrm{C}$ for $48 \mathrm{~h}$. Finally, the acridine orange dye(final concentration $10 \mu \mathrm{g} / \mathrm{mL}$ ) was added. After staining 2-3 minutes, the fluorescence microscope was used to observe the cell apoptosis.

The fourth is to use flow cytometry to detect the effect of BMI1 gene silencing on the apoptosis rate and cell cycle of breast cancer cells. The cells of each group after the action of 5-Fu were digested and collected. And then they were washed two times with pre-cooling PBS. The pre-cooling $70 \%$ ethanol of $-20^{\circ} \mathrm{C}$ was added, and they were fully mixed, and then were preserved at $4^{\circ} \mathrm{C}$ for $24 \mathrm{~h}$. Then they were washed three times with PBS, and the RNaseA (final concentration $10 \mu \mathrm{g} / \mathrm{mL}$ ) was added. Then they were incubated at $37^{\circ} \mathrm{C}$ for $30 \mathrm{~min}$. Finally, the flow cytometer method can be carried out by adding PI (final concentration $50 \mu \mathrm{g} / \mathrm{mL}$ ) and light avoiding staining $30 \mathrm{~min}$. 
Data Statistics. The SPSS16.0 software was used to analyze data. The measurement data is expressed by $\mathrm{x} \pm \mathrm{s}$. The variance analysis was used in the comparison among groups, $\mathrm{t}$ test was used in the comparison of independent two groups, and the significance level of the test was set to 0.05 .

\section{Results}

Inhibitory Effect of BMI1 Gene Silencing on the Proliferation of Breast Cancer Cells. The results of MTT assay showed that BMI1 gene silencing could promote the inhibitory effect of 5-Fu on the growth of breast cancer cells, and with the increase of 5-Fu concentration, the inhibition rate for breast cancer cells increased. The growth inhibition curves of breast cancer cells under different concentrations of 5-Fu were plotted respectively, as shown in Fig.1. It was concluded that the IC50 values of 5-Fu acting for 48h were MCF-7 (2.85 \pm 0.21$)$, MCF-7/BMI1si (1.78 \pm 0.25$)$, MCF-7/5-Fu $(8.05 \pm 0.38)$ and MCF-7/5-Fu/BMI1si $(5.65 \pm 0.27) \mu \mathrm{g} / \mathrm{mL}$, respectively. The IC50 value of BMI1 gene silencing group was significantly lower than that of breast cancer cells MCF-7 and MCF-7/5-Fu(LSD- $t=2.845,3.232 ; P<0.05)$. According to the IC50 value measured by MTT, the subsequent fluorescence observation cell apoptosis and flow cytometry experiment took the $4 \mu \mathrm{g} / \mathrm{mL}$ $5-\mathrm{Fu}$, and $48 \mathrm{~h}$ culture as the parameters of drug action.

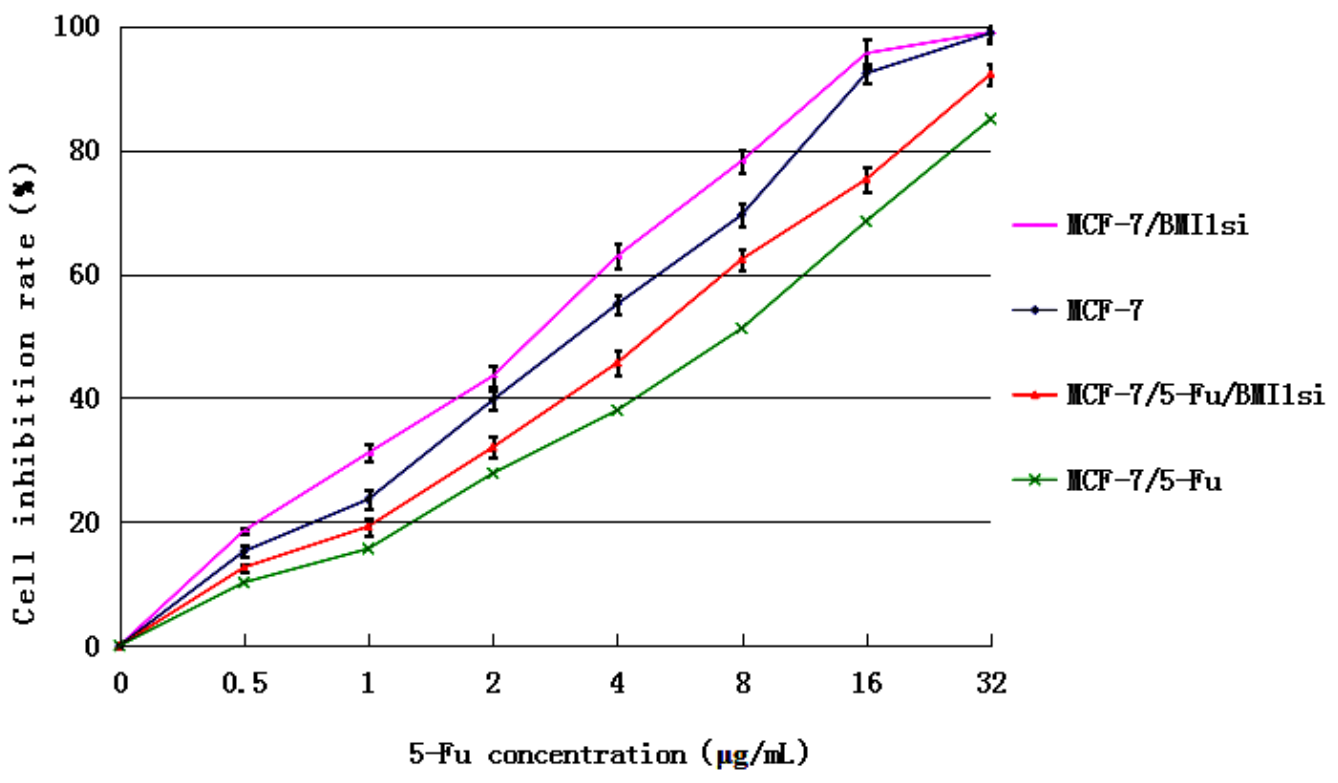

Figure 1. Finite Growth inhibition curve of 5-Fu on cells of each group

BMI1 Gene Silencing Promotes the Morphological Changes of BGC823 Cell Apoptosis. After $48 \mathrm{~h}$ of $4 \mu \mathrm{g} / \mathrm{mL} 5-\mathrm{Fu}$ action, the cells of each group were stained with reactive dye acridine orange(AO), and then were observed under the fluorescence microscope. MCF-7 cells and MCF-7/BMIsi cells, due to the relatively high concentration of drugs, were mainly manifested as the cell necrosis state, and the cells were detached and broken in large area. Some cells of MCF-7/5-Fu cells changed from fusiform shape to ellipse. The surface of the cell membrane was protruding, and many enveloped bodies began to form. The chromatin began to concentrate, initially showing the characteristics of cell apoptosis (see Fif.2). However, the MCF-7/5-Fu/BMI1 si cells shrank, the cell nucleus condensed, vesicles appeared on the surface of cell membrane, forming obvious apoptosis bodies, showing typical apoptosis characteristics(see Fig.3). 


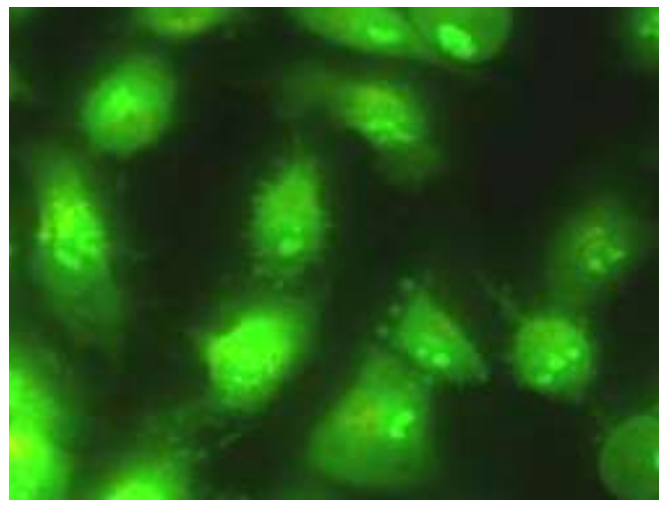

Figure 2. Finite MCF-7/5-Fu cells $(10 \times 40)$

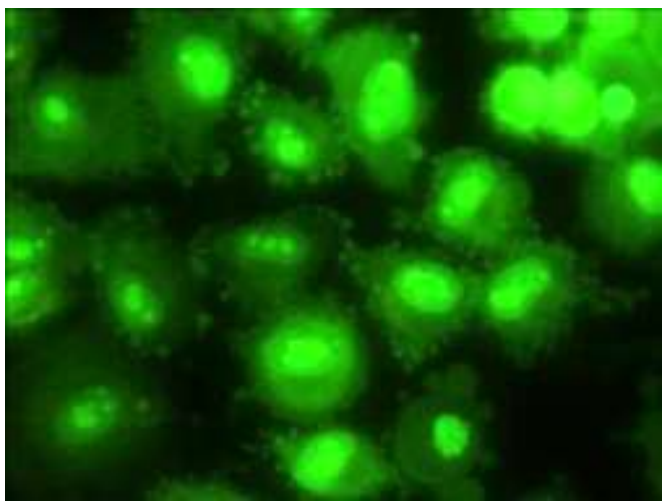

Figure 3. Finite MCF-7/5-Fu/BMI1 si cells $(10 \times 40)$

Effect of BMI1 Gene Silencing on Cell Cycle and Apoptosis Rate of Breast Cancer. In the detection of apoptosis rate, the apoptosis rate of BMI1 gene silencing group was significantly higher than that of MCF-7 and MCF-7/5-Fu of breast cancer cells after the action of $4 \mu \mathrm{g} / \mathrm{mL} 5-\mathrm{Fu}$ for $48 \mathrm{~h}$ (LSD- $t=7.826,8.231 ; P<0.05)$. In the distribution of each phase of cell proliferation cycle, it was found that compared with breast cancer cells MCF-7 and MCF-7/5-Fu, the proportion of cells of the BMI1 gene silencing group in the G0/G1 phase increased significantly (LSD- $t=10.512,8.053$; $P<0.05$ ), however, the proportion of cells in $\mathrm{G}_{2} / \mathrm{M}$ phase decreased significantly (LSD- $t=3.856$, 4.227; $P<0.05$ ), and there was no statistically significant difference in the proportion of cells in $S$ phase (LSD- $t=0.210,0.402 ; P>0.05$ ), as shown in Table 1.

Table 1 Effect of BMI1 gene silencing on cell cycle and apoptosis rate of breast cancer cells

\begin{tabular}{ccccc}
\hline Group & G0/G1 $(\%)$ & $\mathrm{S}(\%)$ & G2/M $(\%)$ & $\begin{array}{c}\text { Apoptosis rate } \\
(\%)\end{array}$ \\
\hline MCF-7 & $36.52 \pm 3.21$ & $24.52 \pm 2.78$ & $39.96 \pm 1.70$ & $41.42 \pm 2.75$ \\
MCF-7/BMI1si & $45.13 \pm 2.01^{*}$ & $25.89 \pm 1.89$ & $28.98 \pm 2.44^{*}$ & $52.26 \pm 1.91^{*}$ \\
\hline $\begin{array}{c}\text { MCF-7/5-Fu } \\
\text { MCF-7/5-Fu/BMI1s } \\
\text { i }\end{array}$ & $23.53 \pm 2.77$ & $26.43 \pm 2.21$ & $50.04 \pm 2.51$ & $22.32 \pm 2.24$ \\
\hline
\end{tabular}

Note: “*”The differences among groups were statistically significant. $(P<0.05)$

\section{Discussions}

The occurrence and development of tumor is a complex process. It is generally believed that the occurrence of tumor is due to the activation of proto oncogenes and the inactivation of anti-oncogenes, which disrupts the normal proliferation, differentiation and apoptosis of cells, 
which is characterized by cell cycle disorder, excessive cell proliferation and poor apoptosis, that is, the imbalance between cell apoptosis and proliferation and cell cycle disorder are the important mechanism for tumorigenesis. Therefore, we can think about the therapeutic strategies of the tumor by two aspects of cell apoptosis and cell cycle. On the one hand, the apoptosis of tumor cells can be induced and promoted to achieve the purpose of inhibiting the proliferation of tumor cells. On the other hand, the tumor cell cycle can be regulated so that the tumor cells can not undergo normal cell division and proliferation.

5-Fluorouracil (5-Fu) is an anti-tumor metabolic drug, which is widely used in the treatment of many solid tumors, such as gastric cancer, colorectal cancer, pancreatic cancer, breast cancer, head and neck cancer and ovarian cancer. It has the characteristics of fast, high efficiency and low toxic side effects. Moreover, 5-Fu is a conventional breast cancer treatment drug. It is commonly used as a first-line clinical drug control for the research of the development of the breast cancer antitumor drug, and its clinical toxicity and drug resistance are typical[11]. BMI1 gene is an important regulatory gene in the polycomb group genes $(\mathrm{PcG})$ family. It participates in the regulation of cell proliferation and differentiation, and plays an important role in maintaining the self renewal and multidirectional differentiation of normal stem cells. The expression level of BMI1 gene is rising as a proto oncogene in many tumors of the human body. Studies have suggested that[12-14] BMI1 plays a role in the regulation of DNA damage response. Through the ubiquitination of histone H2A, it silences the tumor suppressor genes, and it stimulates the ubiquitination of histone $\mathrm{H} 2 \mathrm{~A}$ and $\gamma \mathrm{H} 2 \mathrm{AX}$ to enhance DSBs repair, and reduces the activity of ATM to weaken the activation of cell cycle checkpoint, so that the genetic toxicity and genomic instability increase, thereby participating in the occurrence and evolution of tumors.

In this study, we detected the effect of BMI1 gene silencing on the proliferation and apoptosis of human breast cancer cells through the experiment in vitro. Through the experiment, we found that the chemotherapy drug 5-Fu could inhibit the proliferation of human breast cancer cell MCF-7 and its drug-resistant strain cell, and presented time - dose dependence. BMI1 gene silencing could enhance the sensitivity of breast cancer cells to chemotherapy drug 5-Fu, enhance the effect of inhibiting the proliferation of breast cancer cells, and promote cell apoptosis. According to the experimental results of MTT cell proliferation inhibition, $4 \mu \mathrm{g} / \mathrm{mL} 5$-Fu action was selected for $48 \mathrm{~h}$ to detect the effect of BMI1 gene silencing on the cell cycle and apoptosis rate of breast cancer cells. The results showed that the BMI1 gene silencing increased the proportion of cells in G0/G1 phase, and reduced the proportion of cells in G2/M phase, suggesting that it may have periodic and specific inhibition effect on breast cancer cells. This indicates that BMI1 gene silencing in vitro has a strong inhibitory effect on the proliferation of human breast cancer cell lines, and can enhance the sensitivity of cells to chemotherapy drug 5-Fu, which is consistent with the results of some scholars' research[15-17]. However, the molecular mechanism of the BMI1 gene promoting the development of the tumor remains to be further explored.

In conclusion, BMI1 gene silencing can enhance the sensitivity of human breast cancer cells to chemotherapy drugs, thereby promoting cell apoptosis and blocking cell cycle. It provides an experimental basis for the combination of gene therapy and chemical drugs in the treatment of breast cancer.

\section{Acknowledgement}

Fund project: Funded by the Heilongjiang Natural Science Foundation Project (Project number: C2015033).

\section{References}

[1] Fan L, Strasser-Weippl K, Li JJ, et al. Breast cancer in China [J]. Lancet Oncol, 2014, 15( 7) : 279-289.

[2] Burrows AE, Elledge SJ. How ATR turns on: TopBP1 goes on ATRIP with ATR. Genes 2008; 
Dev. 22, 1416-1421.

[3] Van Lohuizen M, Verbeek S, Scheijen B, et al. Identification ofcooperating oncogenes in E mu-myc transgenic mice by provirus tagging. Cell, 1991;65:737-752.

[4] He X, Dong Y, Wu CW, et al. MicroRNA-218 inhibits cell cycle progression and promotes apoptosis in colon cancer by downregulating BMI1 polycomb ring finger oncogene. Mol Med, 2013;18: 1491-1498.

[5] Bea S, Tort F, Pinyol M, Puig X, Hernandez L, Hernandez S, et al. Bmi-1 gene amplification and overexpression in hematological malignancies occur mainly in mantle cell lymphomas. Cancer Res. 2001; 61: 2409-2412.

[6] Fan C, He L, Kapoor A, Gillis A, Rybak AP, Cutz JC, Tang D. Bmil promotes prostate tumorigenesis via inhibiting p16(INK4a) and p14(ARF) expression. Biochim. Biophys. Acta 2008; 1782: 642-648.

[7] Vonlanthen S, Heighway J, Altermatt HJ, Gugger M, Kappeler A, Borner MM. et al. The Bmi-1 oncoprotein is differentially expressed in non-small cell lung cancer and correlates with INK4a-ARF locus expression. Br. J. Cancer 2001; 84: 1372-1376.

[8] Kim JH, Yoon SY, Kim CN, Joo JH, Moon SK, Choe LS, et al. The Bmi-1 oncoprotein is overexpressed in human colorectal cancer and correlates with the reduced p16INK4a/p14ARF proteins. Cancer Lett. 2004; 203: 217-224.

[9] Kim JH, Yoon SY, Jeong SH, Kim SY, Moon SK, Joo JH, et al. Overexpression of Bmi-1 oncoprotein correlates with axillary lymph node metastases in invasive ductal breast cancer. Breast 2004; 13: 383-388.

[10] Song LB, Zeng MS, Liao WT, Zhang L, Mo HY, Liu WL, et al. Bmi-1 is a novel molecular marker of nasopharyngeal carcinoma progression and immortalizes primary human nasopharyngeal epithelial cells. Cancer Res. 2006; 66: 6225-6232.

[11] Jahani M, Azadbakht M, Norooznezhad F, et al. L- argininealters the effect of 5- fluorouracil on breast cancer cells in favor of apoptosis[J]. Biomed Pharmacother, 2017; 88:114.

[12] Meng X, Wang Y, Zheng X, et al. sh RNA-mediated knockdown of Bmi-1 inhibit lung adenocarcinoma cell migration and metastasis[J]. Lung Cance, 2012; 77( 1) : 24-30.

[13] Chang X, Sun Y, Han S, et al. MiR-203 inhibits melanoma invasive and proliferative abilities by targeting the polycomb group gene BMI1 [J]. Biochem Biophys Res Commun, 2015; 456(1): 361-366.

[14] Bartocci C, Diedrich JK, Ouzounov L, Li J, Piunti A, Pasini D, et al. Isolation of chromatin from dysfunctional telomeres reveals an important role for Ring1B in NHEJ-mediated chromosome fusions. Cell Rep. 2014; 7: 1320-1332.

[15] Cao L, Bombard J, Cintron K, et al. BMI1 as a novel target for drug discovery in cancer[J]. J Cell Biochem, 2011; 112 ( 10 ) :2729-2741.

[16] Li B, Chen H, Wu N, et al. Deregulation of miR-128 in ovarian cancer promotes cisplatin resistance[J]. Int J Gynecol Cancer, 2014; 24(8): 1381-1388.

[17] Nishida Y, Maeda A, Chachad D, et al. Preclinical activity of the novel B-cell-specific Moloney murine leukemia virus integration site 1 inhibitor PTC-209 in acute myeloid leukemia: Implications for leukemia therapy[J]. Cancer Sci, 2015; 106(12): 1705-1713. 